\title{
Self-organizing patterns in an evolutionary rock-paper-scissors game for stochastic synchronized strategy updates
}

\author{
Levente Varga, ${ }^{1,2}$ Jeromos Vukov, ${ }^{1}$ and György Szabó ${ }^{1}$ \\ ${ }^{1}$ Institute of Technical Physics and Materials Science, Research Centre for Natural Sciences, \\ Hungarian Academy of Sciences, P.O. Box 49, H-1525 Budapest, Hungary \\ ${ }^{2}$ Babess-Bolyai University, RO-400084 Cluj-Napoca, Romania
}

(Received 15 August 2014; published 21 October 2014)

\begin{abstract}
We study a spatial evolutionary rock-paper-scissors game with synchronized strategy updating. Players gain their payoff from games with their four neighbors on a square lattice and can update their strategies simultaneously according to the logit rule, which is the noisy version of the best-response dynamics. For the synchronized strategy update two types of global oscillations (with an ordered strategy arrangement and periods of three and six generations) can occur in this system in the zero noise limit. At low noise values, all nine oscillating phases are present in the system by forming a self-organizing spatial pattern due to the comprising invasion and speciation processes along the interfaces separating the different domains.
\end{abstract}

DOI: 10.1103/PhysRevE.90.042920

PACS number(s): 05.45.-a, 02.50.Le, 07.05.Tp, 89.75.Fb

\section{INTRODUCTION}

Game theory gives us a general mathematical framework to study interactive decision situations, when the aims and choices of the players are potentially in conflict [1]. Evolutionary games are introduced to investigate multiagent social and biological systems [2] using the concept of game theory to quantify the interactions. Players can modify their strategy following certain evolutionary rules taking into account Darwinian selection in biological systems and/or individual rationality in social systems. In recent decades, many scientific fields approached game theory; physics proved to be exceptional in handling the spatial systems because of the direct applicability of statistical physics methods (for a survey see $[3,4])$.

Now our investigations are focused on a spatial evolutionary game where pair interactions are described by a cyclic threestrategy game, called the rock-paper-scissors (RPS) game. For the traditional RPS game, players choose simultaneously one of three strategies: rock $\mathbf{R}$, paper $\mathbf{P}$, or scissors $\mathbf{S}$. According to the rules of the game, a strategy is superior to another and inferior to the third one: rock beats scissors, scissors beat paper, and paper beats rock, i.e., the game represents the cyclic dominance of strategies. This type of cyclic dominance plays a crucial role in the maintenance of biodiversity in ecological systems [5-7] and can support the cooperative behavior in social dilemmas when a third strategy (e.g., loners) is present $[3,8,9]$. Similarly, population-level cyclic motions were observed in a human laboratory experiment of the discrete-time iterated RPS game [10]. Moreover, biological experiments have justified the presence of cyclic dominance in populations of bacteria [11-13] and lizards [14,15]. These results have initiated progressive activity to clarify the consequences of cyclic dominance on the macroscopic, populationwide behavior. It is found that these systems exhibit a nontrivial effect when the cyclic symmetries are disturbed [16-19].

Very recently [20] it turned out that all symmetric $3 \times 3$ games can be decomposed as the linear combination of potential and RPS games. For potential games, a potential can be derived from the payoff matrix. The random sequential application of the logit rule drives such systems into a state where the stationary strategy distribution is described by the Boltzmann distribution [21,22]. The RPS game can be considered as the force responsible for driving the system out of equilibrium by generating probability currents (entropy production) throughout the microscopic states of multiagent models [23,24].

For well-mixed populations, systems with RPS-type interactions show global oscillations in the strategy frequencies [25]. For structured populations, i.e., when the interaction network of players is defined by a complex graph, the oscillation can either evolve towards a limit cycle or its amplitude increases continuously until only one strategy (species) remains alive $[26,27]$. On two-dimensional lattices, due to the short-range interactions, numerical simulations have indicated the survival of all three strategies through the formation of self-organizing patterns with rotating spirals [7,28-30]. The general features of the self-organizing patterns (including spatial correlations) have been studied quantitatively in several previous papers [28,31-35]. In contrast to this behavior, in finite one-dimensional systems, investigations indicate domain coarsening [36,37] or fixation for stochastic imitation rules $[33,38,39]$. In three-dimensional lattice systems, on the other hand, vortices (rotating three-edged vertices where the three types of interfaces meet) form closed loops [29,40] in analogy to systems described by the complex Ginzburg-Landau model (see, e.g., [41] and references therein).

Both the complexity of the system and the richness of phenomena increase gradually if we study the effect of cyclic dominance with four [42-51], five [52-56], six [43,57-60], or nine [60-63] strategies. In particular, the studies about ninespecies systems help interpret the results of the present model where one can observe nine ordered spatial arrangements of the three strategies for synchronized strategy update. It is worth mentioning that some of the mentioned three-, four-, and six-species models are subsystems (systems where the use of certain strategies are prohibited) of the nine-species one. In the present model we can explore the competitive relationships among nine strategy associations (which can be identified as complex species) that are built up from three strategies and possess an ordered spatial strategy arrangement. 
It is already well known that the macroscopic behavior of a spatial evolutionary game depends mainly on the number of strategies, the payoff matrix, the connectivity structure, and the dynamical rule(s) controlling the choice of new strategies [3,4,64-66]. The first dynamical rules were introduced to take the Darwinian selection into consideration, allowing the population of the fittest strategy (or species) to grow at the expense of the weaker ones [67,68]. The first spatial evolutionary game was introduced by Nowak and May [69,70] to study the maintenance of cooperative behavior in a lattice model with synchronized strategy update in analogy to cellular automata [71]. In this model, players have modified their strategy at discrete time steps by simultaneously adopting the strategy of the neighbor who received the highest income in the previous step. Subsequently, this deterministic model was modified by the addition of stochastic noise to the imitation process [72]; these rules are used by the followers to study different versions of evolutionary games [73-77]. In the meantime, additional variants of the dynamical rules (including random sequential stochastic updates) were introduced and studied systematically (for a brief survey see $[3,4,64]$ ).

In the literature of physics and economics, a fundamentally different set of dynamical rules was also introduced to take human rationality into account: In the so-called logit rule [22,78-81], players are capable of evaluating their income by testing each available strategy and playing fictitious games against their co-players, assuming the current (quenched) strategy environment. The stochastic logit rule prefers to choose the strategy providing the highest income in an exponential manner. In the low noise limit, it becomes equivalent to the best-response rule [82-84] that may be used to find the Nash equilibria for certain types of games. This rule is intimately related to equilibrium statistical physics because in the case of multiagent potential games, the random sequential applications of the logit rule drive the system into a Boltzmann distribution [22].

In the present work we study an evolutionary RPS game where players are located on the sites of a square lattice with periodic boundary condition. Following one of the three strategies, players play RPS games with their four nearest neighbors in every discrete-time step and after collecting their income, they are allowed to modify their strategy according to a synchronized stochastic logit rule. It will be shown that this system exhibits a curious pattern evolution. When visualizing the spatiotemporal evolution of the strategy distribution, one can observe domains with globally oscillating compositions or structures analogously to the discrete clock models $[85,86]$. In fact, these domains represent different phases of limit cycles and compete against each other via invasions along the interfaces separating them.

The appearance of oscillations within these domains is strongly related to the application of the synchronized logit rule. We have to emphasize that the examination of synchronous activity (e.g., armies marching, churches singing, and communities dancing) has become a promising topic within the social sciences as synchronized actions can increase cooperation and social efficiency [87].

In the next section we describe the model and the technical details of the simulation process. In Sec. III we discuss the results of the model and present and explain the emerging phenomena. In Sec. IV we summarize the main features of the model.

\section{MODEL}

We consider a spatial evolutionary RPS game with synchronized strategy updates at discrete times $t=0,1,2, \ldots$. Players are located on the sites of a square lattice of size $N=L \times L$ and gain their accumulated payoff from games with their nearest neighbors. To facilitate the notation, we use the three-dimensional unit vectors to denote the different strategies, thus the player in the spatial location $x$ can have the strategy vectors

$$
\mathbf{s}_{x}=\mathbf{R} \equiv\left(\begin{array}{l}
1 \\
0 \\
0
\end{array}\right), \quad \mathbf{s}_{x}=\mathbf{S} \equiv\left(\begin{array}{l}
0 \\
1 \\
0
\end{array}\right), \quad \text { or } \mathbf{s}_{x}=\mathbf{P} \equiv\left(\begin{array}{l}
0 \\
0 \\
1
\end{array}\right) .
$$

Using this formalism, the income of player $x$ can be calculated as

$$
\mathbf{u}_{x}\left(\mathbf{s}_{x}, \mathbf{s}_{\Omega_{x}}\right)=\sum_{y \in \Omega_{x}} \mathbf{s}_{x}^{T} \cdot \mathbf{M} \cdot \mathbf{s}_{y},
$$

where $\mathbf{s}_{x}^{T}$ is the transpose of the player's strategy vector and $\mathbf{S}_{\Omega_{x}}$ stands for the strategy profile of players in the interaction neighborhood $\Omega_{x}$ of player $x$, thus the summation runs over the four nearest neighbors of player $x$. The matrix M contains the payoff elements describing the cyclically dominant RPS interactions, namely,

$$
\mathbf{M}=\left(\begin{array}{rrr}
0 & 1 & -1 \\
-1 & 0 & 1 \\
1 & -1 & 0
\end{array}\right)
$$

After players collected their payoff at time $t$, they have the option to adopt a (possibly) more successful strategy according to the evolutionary aspect of the model. In this paper we use the so-called logit rule. In this strategy adoption process players check the payoff they could earn by adopting the possible strategies, provided their neighbors keep using their present strategies. Individuals keep their strategy or adopt a new strategy with probabilities depending on these expectations:

$$
\operatorname{Pr}\left(\mathbf{s}_{x}^{\prime}\right)=\frac{\exp \left[\mathbf{u}_{x}\left(\mathbf{s}_{x}^{\prime}, \mathbf{s}_{\Omega_{x}}\right) / K\right]}{\sum_{\mathbf{s}_{x}^{\prime \prime} \in\{\mathbf{R}, \mathbf{P}, \mathbf{S}\}} \exp \left[\mathbf{u}_{x}\left(\mathbf{s}_{x}^{\prime \prime}, \mathbf{s}_{\Omega_{x}}\right) / K\right]} .
$$

Here $\operatorname{Pr}\left(\mathbf{s}_{x}^{\prime}\right)$ determines the probability for player $x$ to adopt strategy $\mathbf{s}_{x}^{\prime} \in\{\mathbf{R}, \mathbf{P}, \mathbf{S}\}$ where $\mathbf{u}_{x}\left(\mathbf{s}_{x}^{\prime}, \mathbf{s}_{\Omega_{x}}\right)$ is the expected payoff for playing strategy $\mathbf{s}_{x}^{\prime}$ with neighbors who play their present strategies. In addition, $K$ indicates the measure of noise (or temperature) that can originate from different sources and enables the occasional appearance of less successful strategies. The probability of choosing a strategy with smaller expected payoff increases with increasing $K$. Every player decides about the strategy to adopt for the subsequent generation and then the strategy of all players is updated at the same time, i.e., synchronized strategy updating is applied.

We studied the model by performing Monte Carlo simulations on a square lattice with periodic boundary conditions. The size of the lattice varied, depending on the actual problem, from $N=4 \times 10^{4}$ to $10^{6}$. Due to the dynamics and the emerged 
domain structure, these system sizes are large enough to avoid finite-size effect problems. In most of the cases, the simulation is started using random initial conditions where players have equal chance to adopt one of the three different strategies initially; however, to study the invasion phenomena we applied prepared initial states. After a suitable transient time $t_{\mathrm{tr}}$, simulation data are obtained by averaging over a $t_{s}$ sampling time. These parameters are adjusted to the system behavior and are changed typically between $t_{\mathrm{tr}}=1000-5000$ Monte Carlo steps (MCS) and $t_{s}=10^{4}-10^{6} \mathrm{MCS}$, where, within the time unit (MCS), one full strategy updating sequence takes place, i.e., each player modifies strategy once.

\section{RESULTS AND DISCUSSION}

Examining the evolution of the model from a random initial state, we can observe a fast cluster-formation process resulting in domains that exhibit a synchronously oscillating internal structure at sufficiently low noise levels. After some time the domain-growing process stops and the system evolves into a self-organizing spatiotemporal pattern. A typical snapshot is shown in Fig. 1, where nine types of ordered strategy arrangements can be distinguished. Apart from the three obvious homogeneous clusters, there are six more that play an important role during the evolutionary process. These additional clusters have a chessboardlike structure where the dark and light squares of the chessboard are occupied by two different strategies. In fact, for a given strategy pair we can distinguish chessboard and antichessboard patterns where the sublattice occupancy is reversed. Since the sites of the underlying square lattice are identical, chessboard and antichessboard structures may seem to be the same (after all, they are equivalent apart from a translation by one site), but we are going to show that they interact quite differently with other types of clusters, thus they have to be treated as different

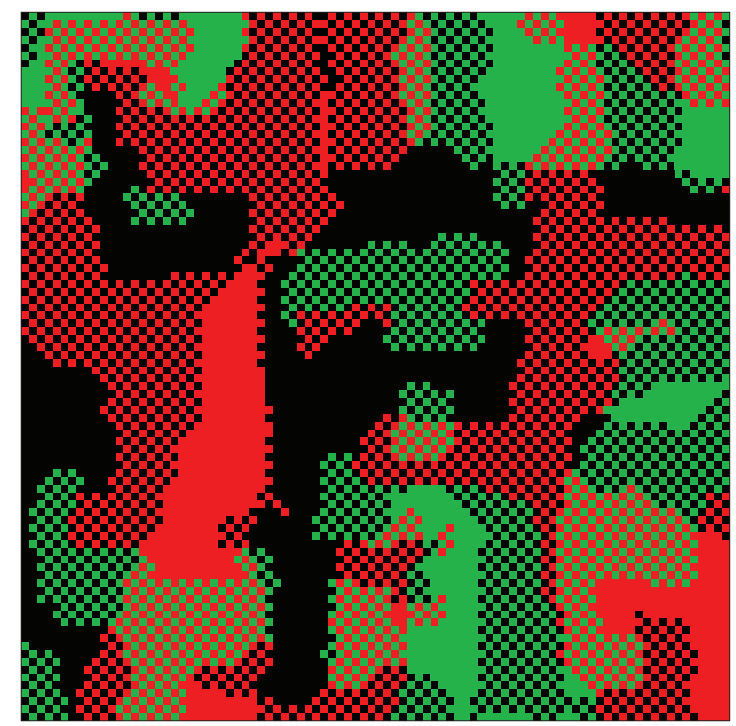

FIG. 1. (Color online) Typical snapshot of the spatial distribution of strategies in a block of $90 \times 90$ sites on a square lattice at $K=0.4$. Black stands for the $\mathbf{R}$ strategy, red (dark gray) for the $\mathbf{P}$ strategy, and green (light gray) for the $\mathbf{S}$ strategy.

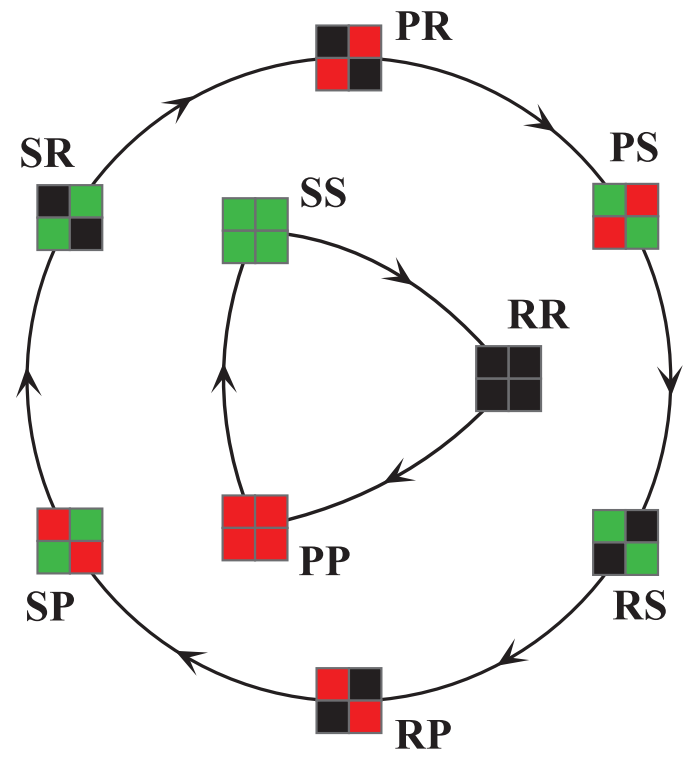

FIG. 2. (Color online) Flow diagram for the cyclic behavior of domains. Solid (black) lines show how the domain composition changes in every generation due to the logit strategy adoption rule. The color code is the same as in Fig. 1. Domain types are denoted by the appropriate strategy names. Note the different names for the chessboard and antichessboard structures. Pure domains return to the same state in three generations; chessboardlike associations accomplish this in six generations. Interestingly, the nine available strategy associations form two disjoint cycles instead of a full cycle.

types as well. We will refer to the different cluster types also as strategy associations further on.

First we discuss what happens inside the homogeneous domains in the limit $K \rightarrow 0$ that corresponds to the synchronized best-response dynamics. Following this myopic rule, players choose the strategy providing the highest income, assuming that co-players do not modify their strategy. If initially $(t=0)$ all players use strategy $\mathbf{R}$, then in the next generation $(t=1)$ everyone will choose $\mathbf{P}$ and subsequently $(t=2) \mathbf{S}$. In short, the homogeneous strategy distribution changes cyclically as illustrated in the inner ring in Fig. 2. These homogeneous states are denoted by the same strategy pair (e.g., SS) positioned on the two sublattices. However, these pairs can be different as well. For example, state PR denotes a sublattice ordered strategy arrangement where the first sublattice is uniformly occupied by $\mathbf{P}$ players and the other one by $\mathbf{R}$ players. Within such a domain, $\mathbf{P}$ players are satisfied and do not wish to modify their strategy, while the dissatisfied $\mathbf{R}$ players are enforced uniformly to apply strategy $\mathbf{S}$, thus the system evolves into the PS state. In this newly formed domain type, $\mathbf{P}$ players are motivated to change their strategy to $\mathbf{R}$ while their co-players keep theirs in the next step. Consequently, one can observe a cyclic behavior that is illustrated in the outer ring in Fig. 2. It is worth mentioning that similar cycles emerge when we consider only two players using the same dynamical rule.

At low noise levels, point defects can appear inside the ordered patterns. These defects, however, vanish in the next generation as the ordered neighborhood dictates the course of evolution. The frequency of isolated point defects increases 


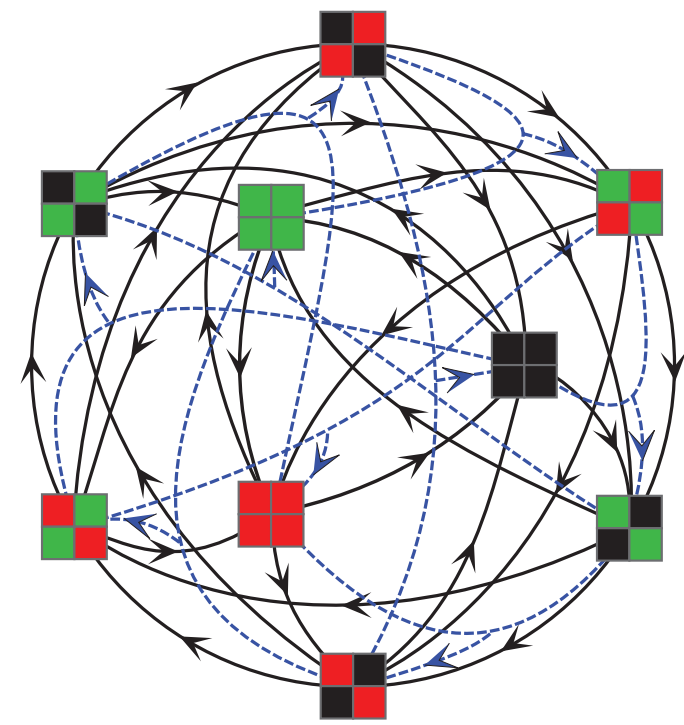

FIG. 3. (Color online) Flow diagram for the invasion and speciation processes between the different strategy associations (domains). Arrows on the solid (black) lines show the winning association when the two associations connected by the black line meet each other. Dashed (blue) lines display the cases when the encounter of two associations results in the appearance of a third species. The arrow points toward the newly established association. The meaning of the strategy colors is the same as in Fig. 2.

gradually with $K$ and for sufficiently high noise levels, a small island of a new phase can appear inside the domains. The resultant dynamics is explored by systematically studying what happens along the interfaces separating different domains.

Figure 3 shows the rather complex, intertwined invasion and speciation relations between the different domain types. Black lines indicate the cases when one of the two clusters connected by the line overcomes the other and invades its territory. The arrow points towards the winning type. The invasion process takes place in a very complicated way as it takes into account the cycles happening in the bulk of the clusters as well. To demonstrate this we have created a scenario with a special initial condition: Figure 4 shows the general layout for an invasion process between two domains of different strategy associations. The two half planes are filled up with different associations ( $\mathbf{R S}$ and $\mathbf{R P}$ ) and their interaction is represented. The first step (indicated by a star) catalyzing the invasion happens as the result of a lower probability event. The green (light gray) $\mathbf{S}$ player appearing in the middle column has an expected payoff of $1\left(P_{S}=1\right)$ instead of the payoff of 2 in the case the $\mathbf{R}$ strategy $\left(P_{R}=2\right)$ was adopted. Thus the probability of the player to indeed adopt the suboptimal strategy is about $0.7 \%$ for $K=0.2$, i.e.,

$$
\operatorname{Pr}(\mathbf{R})=\frac{e^{1 / K}}{e^{1 / K}+e^{2 / K}+e^{-3 / K}} \stackrel{K=0.2}{\approx} 0.0067
$$

This kind of strategy adoption can happen at any place along the domain interface, therefore the overall probability for the starting step of the invasion grows substantially for longer interfaces. As soon as such a steplike irregularity forms, one of the domains can gain ground at the expense of the other. The arrows (and states) following the second panel of Fig. 4 are the result of the highest probability events: The irregularity spreads upward and downward in every generation until the whole column is filled up by the superior domain. Invasion is induced by a (noise-dependent) random event, but after that it continues basically deterministically. To give an idea about the measure of the invasion's noise dependence, we calculated the probability of the abovedescribed steplike irregularity's appearance for two additional $K$ values: $\operatorname{Pr}(\mathbf{R})_{K=0.1}=0.00005$ and $\operatorname{Pr}(\mathbf{R})_{K=0.4}=0.076$. Consequently, for higher noise values, the domain interfaces become rough and the invasion proceeds very fast. On the other hand, in the low noise limit, the domain structure is basically frozen. Similarly to the appearance of the above-described steplike irregularity, other strategies can appear at the domain boundaries as well; however, the likelihood of these events is several magnitudes smaller than that of the event inducing the invasion. The successive panels of the figure demonstrate the six-generation-long cyclic behavior of the domains in a spectacular way.

We have to emphasize that the above invasion process only considered vertical (or horizontal) domain interfaces. For the propagation of diagonal interfaces, no noise-induced starting step is needed. The diagonal interface is essentially a series of steplike irregularities, thus the whole front moves by one site perpendicular to the interface in every generation as the result of the most likely strategy changes.

Besides the black lines in Fig. 3, there are dashed blue lines connecting three strategy associations. These dashed lines indicate cases when the encounter of two domains gives birth to a third type of cluster that consumes the first two. The arrow points towards this newly established domain type. This process can also be considered as speciation: The encounter of two species (associations) generates a third one. The actual course of speciation takes place similarly to that of invasion: The third species forms at the interface and then it consumes both original associations according to the scenario described in Fig. 4. We illustrate this process in Fig. 5 on a larger scale.
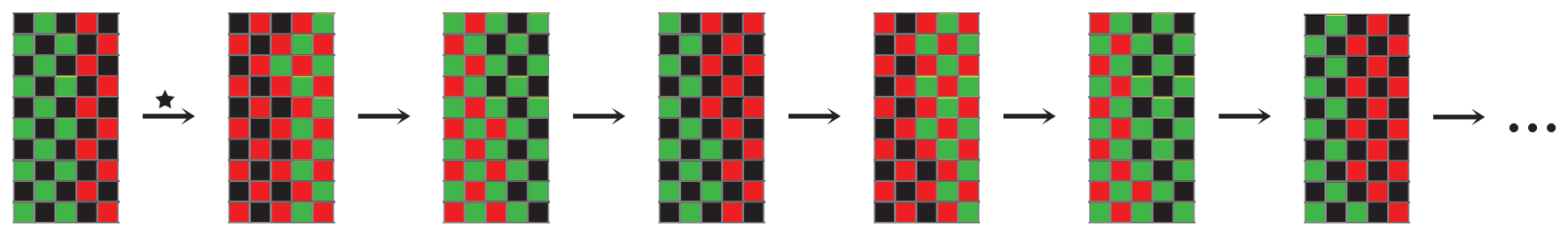

FIG. 4. (Color online) Propagation of a steplike irregularity at the boundary of two domains. The first step of the invasion (indicated by a star) happens with a lower noise-dependent probability. The propagation of the irregularity displayed on the subsequent panels is, however, the result of the most probable strategy adoptions. The six-generation-long cyclic behavior of the domains can be observed as well. 

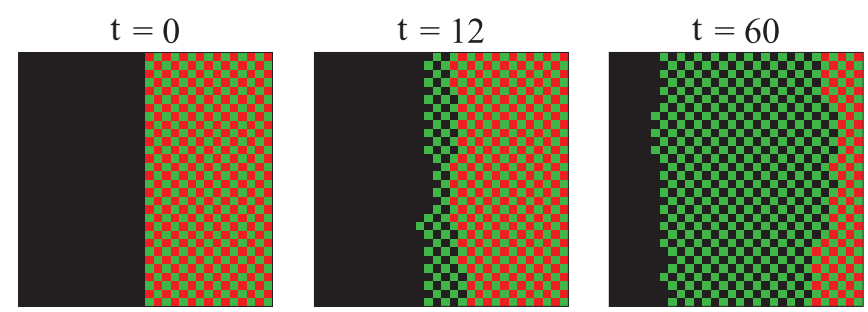

FIG. 5. (Color online) Snapshots showing the course of a speciation event at $K=0.3$. The new species $\mathbf{S R}$ emerges at the boundary of the $\mathbf{R R}$ and $\mathbf{S P}$ domains.

In some cases, the invasion process goes on in a slightly different way. A third type of strategy association can form at the interface, however, in this instance, this new species does not emerge victoriously; it only helps one of the domains to invade the other. It pushes forward into the territory of the inferior domain and at the same time the superior domain incorporates its territory from the other side. As a result, the new species acts as a catalyst for invasion in a narrow strip and as soon as the region of the inferior domain is consumed, this new species is annihilated by the superior domain as well.

For the quantitative investigation of the spatial patterns we have determined the autocorrelation function $c(z)$ that measures the probability of finding strategy $\mathbf{R}$ at two sites with a horizontal (or vertical) distance $z$ between the sites. Namely,

$$
c(z)=\left\langle n_{x}(\mathbf{R}) n_{x+z}(\mathbf{R})\right\rangle_{x, t}-\left\langle n_{x}(\mathbf{R})\right\rangle_{x, t}^{2},
$$

where $\langle\cdots\rangle_{x, t}$ denotes averaging over the lattice sites $x$ and time $t$ in the stationary state and the occupation number $n_{x}(\mathbf{R})=1$ if $\mathbf{s}_{x}=\mathbf{R}$ and $n_{x}(\mathbf{R})=0$ otherwise. Evidently, similar correlation functions can be defined for the other two strategies, however, these quantities are equivalent due to the cyclic symmetries.

As the spatial pattern is a mixture of homogeneous and chessboardlike domains, an oscillation (with a period of 2) can be observed in the autocorrelation function where $c(z)$ tends to zero with a different speed at the odd and even values of $z$. At high noise values, the correlations vanish exponentially. In contrast, at low noise level, a suppressed oscillation can be observed in $c(z)$ at even distances as illustrated in Fig. 6 for $K=0.3$ and 0.4 . The estimated value where $c(z)$ becomes zero is proportional to the average linear domain size that increases when the noise is decreased. The quantitative analysis of the system's behavior in the low noise limit is prevented by technical difficulties related to the extremely slow motion of interfaces.

\section{SUMMARY}

We have studied an evolutionary RPS game with myopic synchronized strategy updates on a square lattice. We

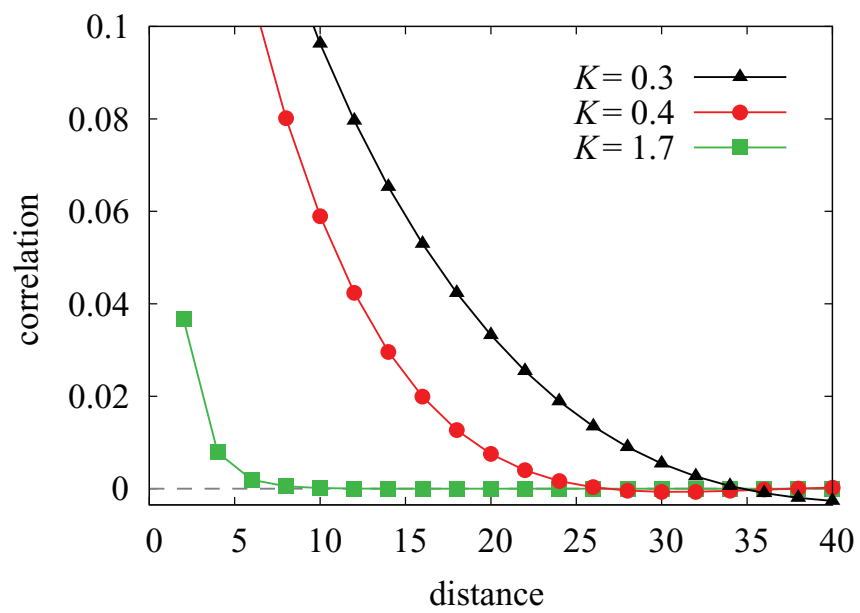

FIG. 6. (Color online) Autocorrelation function versus distance of even integers for three different noise levels. Dashed line shows the zero level. The system size used in the simulations was $400 \times 400$.

observed peculiar self-organizing patterns including invasion and speciation processes. One of the striking features of these globally oscillating patterns is that we can distinguish domains representing ordered phases of the three- or six-steplong limit cycles. These limit cycles can be considered as oscillating solutions consisting of strategy associations with spatiotemporal structure governing the system's behavior at small sizes and low noise levels. The self-organizing patterns emerge in a sufficiently large system if it is started from a random initial state.

The self-organizing pattern formation of these domains is maintained by invasions between them. The direction of invasion is determined by the RPS-type competitions between the components of the oscillating domains. It is found that this direction is preserved during the consecutive steps. The invasion directions reflect symmetries that are assumed in models of bacterial warfare where bacteria use two toxins (and antitoxins) during their evolutionary competition. Besides the cyclic invasion phenomena we could observe speciation processes when a new phase emerges and extends along the interfaces of the original domains. All these features are summarized in an extended flow diagram characterizing the invasion and speciation processes between the strategy associations.

\section{ACKNOWLEDGMENTS}

This research was supported by the John Templeton Foundation (FQEB Grant No. RFP-12-22), the Hungarian Scientific Research Fund (OTKA Grant No. TK-101490), and the European Social Fund through project FutureICT.hu (No. TÁMOP-4.2.2.C-11/1/KONV-2012-0013).
[1] J. von Neumann and O. Morgenstern, Theory of Games and Economic Behaviour (Princeton University Press, Princeton, 1944).

[2] J. Maynard Smith, Evolution and the Theory of Games (Cambridge University Press, Cambridge, 1982).
[3] M. A. Nowak, Evolutionary Dynamics (Harvard University Press, Cambridge, 2006).

[4] G. Szabó and G. Fáth, Phys. Rep. 446, 97 (2007).

[5] R. M. May and W. J. Leonard, SIAM J. Appl. Math. 29, 243 (1975). 
[6] J. Hofbauer and K. Sigmund, The Theory of Evolution and Dynamical Systems (Cambridge University Press, Cambridge, 1988).

[7] T. Reichenbach, M. Mobilia, and E. Frey, Nature (London) 448 , 1046 (2007).

[8] C. Hauert, S. De Monte, J. Hofbauer, and K. Sigmund, Science 296, 1129 (2002).

[9] K. Sigmund, The Calculus of Selfishness (Princeton University Press, Princeton, 2010).

[10] Z. Wang, B. Xu, and H.-J. Zhou, Sci. Rep. 4, 5830 (2014).

[11] B. Kerr, M. A. Riley, M. W. Feldman, and B. J. M. Bohannan, Nature (London) 418, 171 (2002).

[12] B. C. Kirkup and M. A. Riley, Nature (London) 428, 412 (2004).

[13] E. Frey, Physica A 389, 4265 (2010).

[14] B. Sinervo and C. M. Lively, Nature (London) 380, 240 (1996).

[15] C. Guill, B. Drossel, W. Just, and E. Carmack, J. Theor. Biol. 276, 16 (2011).

[16] K. Tainaka, Phys. Lett. A 176, 303 (1993).

[17] K. Tainaka, Phys. Lett. A 207, 53 (1995).

[18] R. Durrett and S. Levin, J. Theor. Biol. 185, 165 (1997).

[19] M. Frean and E. D. Abraham, Proc. R. Soc. London Ser. B 268, 1323 (2001).

[20] G. Szabó, K. S. Bodó, B. Allen, and M. A. Nowak, Phys. Rev. E 90, 042811 (2014).

[21] D. Monderer and L. S. Shapley, Games Econ. Behav. 14, 124 (1996).

[22] L. E. Blume, Games Econ. Behav. 5, 387 (1993).

[23] J. Schnakenberg, Rev. Mod. Phys. 48, 571 (1976).

[24] B. Andrae, J. Cremer, T. Reichenbach, and E. Frey, Phys. Rev. Lett. 104, 218102 (2010).

[25] J. Hofbauer and K. Sigmund, Evolutionary Games and Population Dynamics (Cambridge University Press, Cambridge, 1998).

[26] G. Szabó, A. Szolnoki, and R. Izsák, J. Phys. A: Math. Gen. 37, 2599 (2004).

[27] A. Szolnoki and G. Szabó, Phys. Rev. E 70, 037102 (2004).

[28] K. Tainaka and Y. Itoh, Europhys. Lett. 15, 399 (1991).

[29] K.-I. Tainaka, Phys. Rev. E 50, 3401 (1994).

[30] G. Szabó and A. Szolnoki, Phys. Rev. E 65, 036115 (2002).

[31] A. Szolnoki and G. Szabó, Phys. Rev. E 70, 027101 (2004).

[32] A. Szolnoki, G. Szabó, and M. Ravasz, Phys. Rev. E 71, 027102 (2005).

[33] M. Mobilia, I. T. Georgiev, and U. C. Täuber, Phys. Rev. E 73, 040903(R) (2006).

[34] M. Peltomäki, M. Rost, and M. Alava, J. Stat. Mech. (2009) P02042.

[35] J. Juul, K. Sneppen, and J. Mathiesen, Phys. Rev. E 87, 042702 (2013).

[36] L. Frachebourg, P. L. Krapivsky, and E. Ben-Naim, Phys. Rev. E 54, 6186 (1996).

[37] L. Frachebourg, P. L. Krapivsky, and E. Ben-Naim, Phys. Rev. Lett. 77, 2125 (1996).

[38] L. Frachebourg and P. L. Krapivsky, J. Phys. A 31, L287 (1998).

[39] J. C. Claussen and A. Traulsen, Phys. Rev. Lett. 100, 058104 (2008).

[40] K.-I. Tainaka, Phys. Rev. Lett. 63, 2688 (1989).

[41] S. Mowlaei, A. Roman, and M. Pleimling, J. Phys. A: Math. Theor. 47, 165001 (2014).

[42] G. Szabó and G. A. Sznaider, Phys. Rev. E 69, 031911 (2004).

[43] G. Szabó, J. Phys. A: Math. Gen. 38, 6689 (2005).
[44] T. Reichenbach and E. Frey, Phys. Rev. Lett. 101, 058102 (2008).

[45] M. Peltomäki and M. Alava, Phys. Rev. E 78, 031906 (2008).

[46] Q. He, M. Mobilia, and U. C. Täuber, Phys. Rev. E 82, 051909 (2010).

[47] C. H. Durney, S. O. Case, M. Pleimling, and R. K. P. Zia, Phys. Rev. E 83, 051108 (2011).

[48] A. Roman, D. Konrad, and M. Pleimling, J. Stat. Mech. (2012) P07014.

[49] A. F. Lütz, S. Risau-Gusman, and J. J. Arenzon, J. Theor. Biol. 317, 286 (2013).

[50] B. Intoy and M. Pleimling, J. Stat. Mech. (2013) P08011.

[51] A. Dobrinevski, M. Alava, T. Reichenbach, and E. Frey, Phys. Rev. E 89, 012721 (2014).

[52] R. A. Laird and B. S. Schamp, J. Theor. Biol. 256, 90 (2009).

[53] P. P. Avelino, D. Bazeia, L. Losano, and J. Menezes, Phys. Rev. E 86, 031119 (2012).

[54] Y. Kang, Q. Pan, X. Wang, and M. He, Physica A 392, 2652 (2013).

[55] J. Vukov, A. Szolnoki, and G. Szabó, Phys. Rev. E 88, 022123 (2013).

[56] P. P. Avelino, D. Bazeia, J. Menezes, and B. F. de Oliveira, Phys. Lett. A 378, 393 (2014).

[57] G. Szabó and T. Czárán, Phys. Rev. E 64, 042902 (2001).

[58] R. A. Laird and B. S. Schamp, Am. Nat. 168, 182 (2006).

[59] M. Perc, A. Szolnoki, and G. Szabó, Phys. Rev. E 75, 052102 (2007).

[60] A. Roman, D. Dasgupta, and M. Pleimling, Phys. Rev. E 87, 032148 (2013).

[61] G. Szabó and T. Czárán, Phys. Rev. E 63, 061904 (2001).

[62] P. Szabó, T. Czárán, and G. Szabó, J. Theor. Biol. 248, 736 (2007).

[63] R. A. Laird and B. S. Schamp, Ecology 89, 237 (2008).

[64] C. P. Roca, J. A. Cuesta, and A. Sánchez, Phys. Life Rev. 6, 208 (2009).

[65] M. Perc and A. Szolnoki, BioSystems 99, 109 (2010).

[66] M. Perc, J. Gómez-Gardeñes, A. Szolnoki, L. M. Floría, and Y. Moreno, J. R. Soc. Interface 10, 20120997 (2013).

[67] J. Maynard Smith and G. R. Price, Nature (London) 246, 15 (1973)

[68] R. Axelrod and W. D. Hamilton, Science 211, 1390 (1981).

[69] M. A. Nowak and R. M. May, Nature (London) 359, 826 (1992).

[70] M. A. Nowak and R. M. May, Int. J. Bifurcat. Chaos 03, 35 (1993).

[71] S. Wolfram, Rev. Mod. Phys. 55, 601 (1983).

[72] M. A. Nowak, S. Bonhoeffer, and R. M. May, Int. J. Bifurcat. Chaos 04, 33 (1994).

[73] K. Lindgren and M. G. Nordahl, Physica D 75, 292 (1994).

[74] C. Hauert, Proc. R. Soc. London Ser. B 268, 761 (2001).

[75] R. Alonso-Sanz, C. Martín, and M. Martín, Int. J. Bifurcat. Chaos 11, 2037 (2001).

[76] G. Abramson and M. Kuperman, Phys. Rev. E 63, 030901(R) (2001).

[77] J. Alonso, A. Fernández, and H. Fort, J. Stat. Mech. (2006) P06013.

[78] L. E. Blume, Games Econ. Behav. 11, 111 (1995).

[79] D. Fudenberg and D. Levine, The Theory of Learning in Games (MIT Press, Cambridge, 1998).

[80] J. Hofbauer and W. H. Sandholm, J. Econ. Theor. 132, 47 (2007). 
[81] W. H. Sandholm, Population Games and Evolutionary Dynamics (MIT Press, Cambridge, 2010).

[82] G. W. Brown, in Activity Analysis of Production and Allocation, edited by T. C. Koopmans (Wiley, New York, 1951), pp. 373-376.

[83] D. Fudenberg and J. Tirole, Game Theory (MIT Press, Cambridge, 1991).
[84] R. Gibbons, Game Theory for Applied Economists (Princeton University Press, Princeton, 1992).

[85] Z. Néda, E. Ravasz, T. Vicsek, Y. Brechet, and A. L. Barabasi, Phys. Rev. E 61, 6987 (2000).

[86] Z. Néda, A. Nikitin, and T. Vicsek, J. Phys. A 321, 238 (2003).

[87] S. S. Wiltermuth and C. Heath, Psychol. Sci. 20, 1 (2009). 